\title{
Cerebral Proliferative Angiopathy: An Uncommon and Misdiagnosed Entity
}

\author{
Sarbesh Tiwari ${ }^{1}$ Pawan K. Garg ${ }^{1}$ Pushpinder S. Khera ${ }^{1}$ Santhosh Babu ${ }^{1}$ Binit Sureka ${ }^{1}$ Taruna Yadav ${ }^{1}$ \\ ${ }^{1}$ Department of Diagnostic and Interventional Radiology, All India \\ Address for correspondence Pawan Kumar Garg, MD, Department \\ Institute of Medical Sciences, Basni, Jodhpur, Rajasthan, India \\ of Diagnostic and Interventional Radiology, All India Institute of \\ Medical Sciences, Basni, Jodhpur 342005, Rajasthan, India \\ (e-mail: drgargpawan@gmail.com).
}

\begin{abstract}
Keywords

- cerebral proliferative angiopathy

- arteriovenous

malformation

Cerebral proliferative angiopathy (CPA), previously known as diffuse nidus-type arteriovenous malformation (AVM) is an entity distinct from cerebral AVM, characterized by multiple small arterial feeders, large-size nidus involving entire lobe or hemisphere, and no early draining veins with normal brain parenchyma interspersed between the abnormal vessels. It is usually seen in younger age group and is more common in females. We hereby report a case of diffuse cerebral proliferative angiopathy in a 29-year-old man who presented with intracranial hemorrhage. It is important to recognize this entity to avoid aggressive treatment, thus preventing permanent damage to the normal intermingled brain tissue.
\end{abstract}

\section{Introduction}

Cerebral proliferative angiopathy (CPA) is a type of vascular malformation of brain with symptoms and imaging appearance similar to arteriovenous malformation (AVM). However, multiple features help in diagnosis of CPA on digital subtraction angiography (DSA) including diffuse network of vessels with intermingled normal brain parenchyma, large nidus size, no or minimal hypertrophy of feeding arteries, absence of flow-related aneurysms, absence of large draining vein, and diffuse angiogenesis.

\section{Case Report}

A 29-year-old man presented to neurosurgery outpatient department (OPD) of an outside hospital with chronic unsteadiness of gait with sudden onset of headache and weakness in right upper and lower limb. His Glasgow Coma Scale (GCS)score was $15 / 15$ at the time of presentation. There was no history of hypertension or diabetes. Other laboratory investigations were unremarkable. Noncontrast

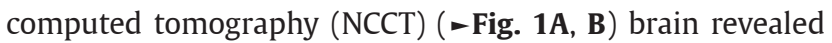
small amount of intraventricular hemorrhage with some hyperdense vascular channels in left temporal lobe. Considering possibility of vascular malformation, computed tomography (CT) angiography of brain was performed using $50 \mathrm{~mL}$

published online Feb 4, 2020
DOI https://doi.org/

10.1055/s-0039-3401329

ISSN 2457-0214. intravenous contrast iohexol (Contrapaque, J.B. Chemicals) on a 256-slice dual-source CT scanner (Siemens Somatom Flash, Siemens $\mathrm{GmBH}$ ) which revealed an abnormal tangle of vessels in left temporal lobe consistent with a nidus of AVM being supplied by branches of left middle and posterior cerebral arteries; however, no hypertrophy of feeder arteries was noted. (-Fig. 1C).

Considering the possibility of a large ruptured cerebral AVM a digital subtraction angiography (DSA) of cerebrovertebral arteries was performed for further characterization, to get dynamic information and to look for risk factors like intranidal aneurysm and venous sac. The study was performed under local anesthesia through right transfemoral route on a biplane DSA machine (Allura Clarity FD 20/20, Philips) using iohexol as intravenous (IV) contrast. A 5-Fr, 11-cm angiography sheath (Cook Medical) was placed in the right femoral artery after accessing the artery under fluoroscopic guidance using a $18 \mathrm{G}$ puncture needle (Cook Medical). Selective angiographic runs were obtained using a 5-Fr H1 diagnostic catheter (Cook Medical) for bilateral internal carotid arteries (ICA), external carotid arteries (ECA) and vertebral arteries.

Left ICA angiogram ( - Fig. 2A, B) revealed a large sized diffuse nidus involving the entire left temporal lobe being supplied by multiple small branches of left middle cerebral arteries with no significant arterial hypertrophy. The nidus was also supplied 

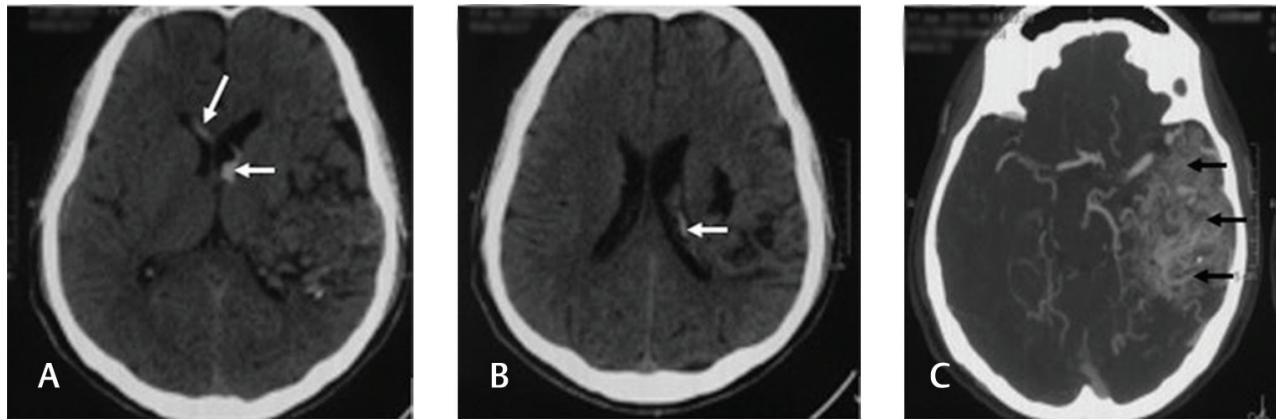

Fig. 1 Noncontrast computed tomography (NCCT) head (A, B) reveal intraventricular hemorrhage in frontal horn of bilateral and body of left lateral ventricle (white arrow). On computed tomography angiography (C) there is presence of large tangle of vessels (black arrow) suggestive of nidus in left temporal lobe.
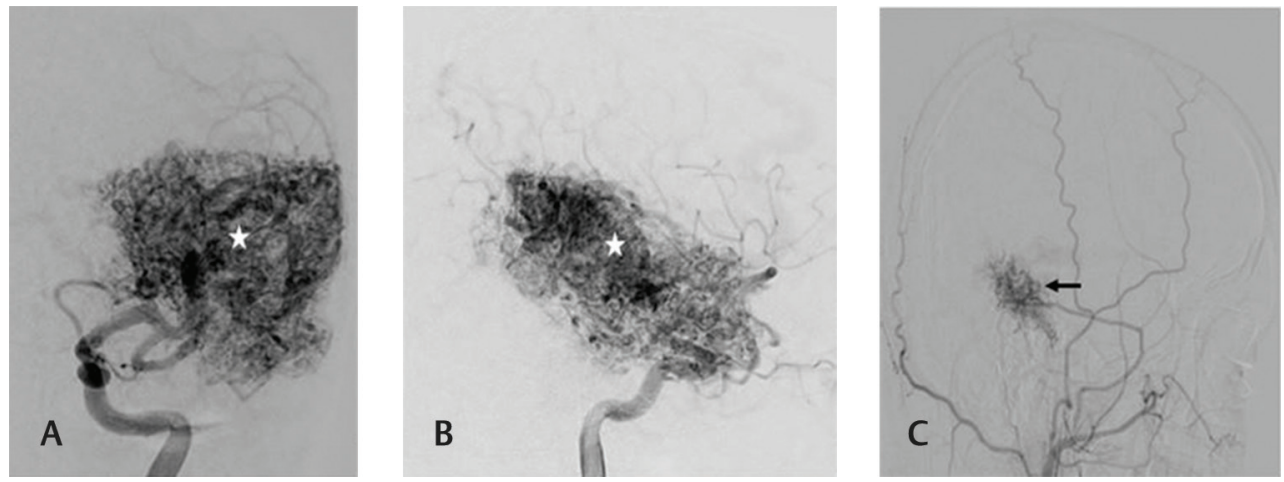

Fig. 2 Digital substraction angiography (DSA) anteroposterior (A) and lateral (B) view from left internal carotid artery injection showing large nidus (asterisk) in left temporal region with no intranidal aneurysm, no early draining vein, or venous sac. Middle cerebral artery branches supplying nidus is not much hypertrophied. Left external carotid artery injection (C) lateral view reveal transdural supply (black arrow) to the nidus.
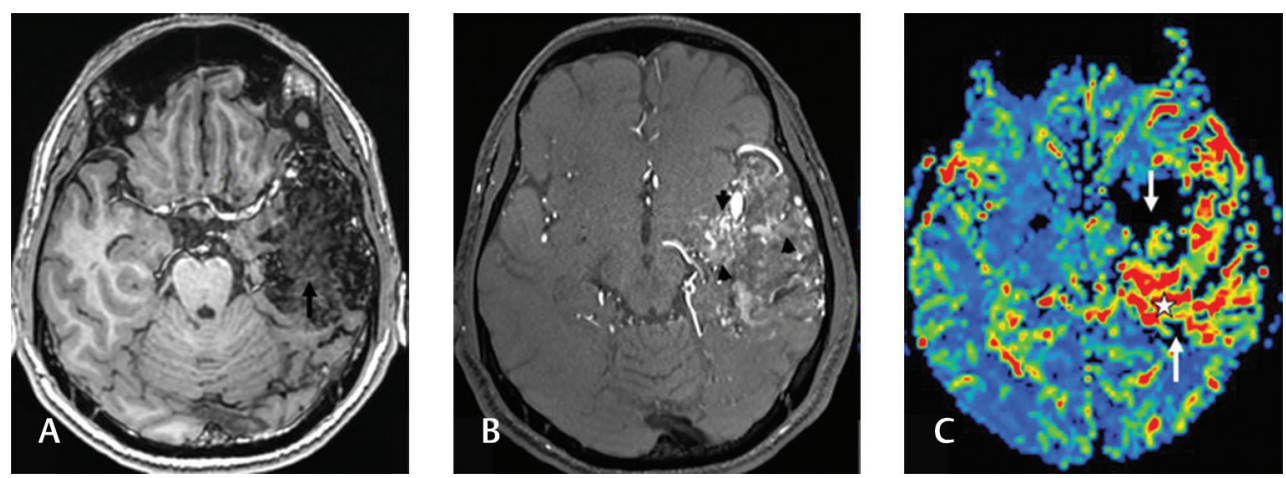

Fig. 3 Magnetic resonance imaging (MRI) brain T1W axial image (A) showing flow voids in left temporal lobe with intervening normal brain parenchyma (black arrow). Magnetic resonance (MR) angiography (B) reveals presence of tangles of vessels to suggest nidus (short black arrows). (C) MR perfusion images show increased cerebral blood volume (CBV) in the nidus (asterisk) with decreased CBV in surrounding parenchyma (white arrow).

by small branches of left posterior cerebral artery. Transdural feeders from left ECA branches ( - Fig. 2C) were also seen to be supplying the nidus. No intranidal aneurysms or venous sac and early draining vein were identified. Considering all these features a possibility of CPA was thought of and further magnetic resonance imaging (MRI) with perfusion study was performed.

MRI brain was performed on a 3 Tesla scanner (Discovery HD, General Electric, Milwaukee, United States). MRI brain

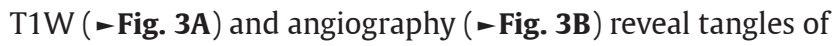

vessels in left temporal lobe with normal interspersed brain parenchyma. MR perfusion ( - Fig. 3C) performed which reveal increased cerebral blood volume (CBV) in nidus. The $\mathrm{CBV}$ is decreased in parenchyma around the nidus. These features further favor a diagnosis of CPA, since its etiology is thought to be due to abnormal angiogenesis in response to cortical ischemia and widespread hypoperfusion.

As there is normal interspersed brain parenchyma, aggressive treatment in form of endovascular embolization, 
radiotherapy or surgery was not advised, since literature review suggested that it might lead to loss of blood supply to interspersed brain parenchyma leading to ischemia and worsening of symptoms. Patient was advised medical management with antiepileptics and regular imaging follow-up.

\section{Discussion}

Vascular lesions or malformations of brain include a wide variety of disorders with overlapping imaging and clinical features. The commonest classification system divides vascular lesions into AVM, which may be either pial or dural (depending on the location of the shunt); cavernous hemangiomas (or cavernomas); capillary telangiectasias; and developmental venous anomalies (DVAs, formerly known as venous angiomas). ${ }^{1}$

Cerebral proliferative angiopathy (CPA), previously known as diffuse nidus type arteriovenous malformation (AVM) ${ }^{1} \mathrm{CPA}$ is a distinct entity different from cerebral AVM in angioarchitecture, natural history, clinical presentation, and, therefore, treatment options. ${ }^{2}$ It is characterized by a diffuse network of vessels with intermingled normal brain parenchyma. Large size of the nidus and the small shunting volume, no or minimal hypertrophy of feeding arteries, absence of flow-related aneurysms, absence of large draining vein and venous sac, diffuse angiogenesis (e.g., transdural supply, progressive arterial occlusion) are the angiographic hallmarks of this disease. ${ }^{1-3}$ Parenchymal involvement often involves an entire lobe or even a hemisphere with the nidus fed by multiple normal or moderately enlarged arteries and associated stenosis of feeder arteries. ${ }^{2,3}$

Patients with this disease present with intractable seizures, motor deficits, headaches and stroke like symptoms. Hemorrhage is relatively uncommon. ${ }^{1,4}$ However, in patients complicated with bleeding, the estimated risk of rebleeding is high (67\%). ${ }^{4}$ This condition most commonly affects women of young age group, in a ratio of 2:1 and is reported as a rare entity. It corresponds to $3.4 \%$ of all cerebral arteriovenous malformations. ${ }^{15,6}$

CT or MRI usually shows diffuse vascular lesions with interspersed normal brain parenchyma. MR perfusion is helpful, it shows increased cerebral blood volume (CBV) and only slightly decreased time to peak (TTP) and a prolonged mean transit time (MTT) in the nidus. Regions of increased TTP values and decreased CBV located remote from the nidus, in both cortical and subcortical areas are indicative of remote and widespread hypoperfusion. ${ }^{1}$

This cerebral hypoperfusion in CPA is evaluable by using $\mathrm{N}$-isopropyl-p- $\left[{ }^{123} \mathrm{I}\right]$ iodoamphetamine single-photon emission computed tomography ( ${ }^{123}$ I-IMP-SPECT). ${ }^{123}$ I-IMP-SPECT at resting state shows preserved uptake within the vascular lesion, yet lower uptake in the area adjacent to the lesion suggests cerebral ischemia. ${ }^{7}$

DSA is gold standard for diagnosis of CPA due to its realtime dynamic flow evaluation capability. The DSA generally shows a nidus size greater than $6 \mathrm{~cm}$ with venous drainage not having ectasia, in most cases. ${ }^{8}$
If histopathology is done, the lesion shows abnormal arteries and veins with altered lamination of the internal elastic lamina and muscle fibers on the arterial site and collagenous thickening of the veins. ${ }^{1}$

Due to presence of viable cerebral parenchyma interspersed between the tangled nidus, attempts to treat these patient by any measure like surgery, embolization, or radiotherapy can aggravate the neurological deficits. However, therapeutic management is indicated in extreme cases, such as intractable epileptic seizures although with great risks of aggravating neurological deficits. Since the pathomechanism is ischemia-induced angiogenesis, successful treatment with pial synangiosis or burr-hole therapy to enhance supply to healthy brain tissue from the external carotid artery are suggested (similar to Moyamoya disease). ${ }^{1,9}$ Encephaloduroarteriosynangiosis (EDAS) has been described to improve symptoms in adult CPA with cerebral ischemia in which neurological deficits. ${ }^{9}$

Evidence of ongoing angiogenesis with elevated levels of vascular endothelial growth factor (VEGF) basic fibroblast growth factor in CSF has been described in patients with CPA; however, it is currently not clear if treatment with monoclonal antibody against VEGF could be of benefit. ${ }^{5,10}$

\section{Conclusion}

Cerebral proliferative angiopathy is a rare distinct entity which should be differentiated from classic cerebral AVM. Aggressive therapeutic treatment in the form of endovascular embolization, surgery, or radiotherapy may lead to permanent brain parenchymal damage and aggravate neurological deficits. Since the pathomechanism of CPA is ischemia-induced angiogenesis, treatment in the form of pial synangiosis or burr-hole therapy may be successful. In future monoclonal antibody against various growth factors may be useful to treat CPA.

\section{Source(s) of Support}

None.

\section{Presentation at a Meeting}

None.

\section{Conflics of Interest}

None.

\section{Acknowledgments}

None.

\section{References}

1 Geibprasert S, Pongpech S, Jiarakongmun P, Shroff MM, Armstrong DC, Krings T. Radiologic assessment of brain arteriovenous malformations: what clinicians need to know. Radiographics 2010;30(2):483-501

2 Lasjaunias PL, Landrieu P, Rodesch G, et al. Cerebral proliferative angiopathy: clinical and angiographic description of an entity different from cerebral AVMs. Stroke 2008;39(3):878-885

3 Maekawa H, Tanaka M, Hadeishi H. Fatal hemorrhage in cerebral proliferative angiopathy. Interv Neuroradiol 2012;18(3):309-313 
4 Bilaj F, Rroji A, Enesi E, Ruka M, Petrela M. Cerebral proliferative angiopathy with tumor-like hemorrhage: a case report and literature review. Neuroradiol J 2016;29(5):336-339

5 Gold JJ, Crawford JR. Acute hemiparesis in a child as a presenting symptom of hemispheric cerebral proliferative angiopathy. Case Rep Neurol Med 2013;2013:920859

6 Khan A, Rattihalli R, Beri S, Dickinson F, Hussain N, Gosalakkal J. Cerebral proliferative angiopathy: a rare form of vascular malformation. J Paediatr Child Health 2013;49(6):504-505

7 Kimiwada T, Hayashi T, Shirane R, Tominaga T. 123I-IMP-SPECT in a patient with cerebral proliferative angiopathy: a case report. J Stroke Cerebrovasc Dis 2013;22(8):1432-1435
8 Lv X, Wu Z, Jiang C, et al. Illustrative case: a patient with cerebral proliferative angiopathy. Eur J Radiol Extra 2011;78:e67-e70

9 Kono K, Terada T. Encephaloduroarteriosynangiosis for cerebral proliferative angiopathy with cerebral ischemia. J Neurosurg 2014;121(6):1411-1415

10 Marks MP, Steinberg GK. Cerebral proliferative angiopathy. J Neurointerv Surg 2012;4(5):e25 